\title{
BMJ Open Moxibustion versus diclofenac sodium gel for the treatment of knee osteoarthritis: a study protocol for a double-blinded, double-placebo, randomised controlled trial
}

Jian-Ying Zhou, Ling Luo, Lin-Lin Zhu, Hai-yan Yin, Qiaofeng Wu, Jia-xi Peng, Cheng-shun Zhang, Peng Lv, Yong Tang, Shu-guang Yu

To cite: Zhou J-Y,

Luo L, Zhu L-L, et al. Moxibustion versus diclofenac sodium gel for the treatment of knee osteoarthritis: a study protocol for a double-blinded, double-placebo, randomised controlled trial. BMJ Open 2017;7:e012879. doi:10.1136/ bmjopen-2016-012879

- Prepublication history and additional material for this paper are available online. To view these files please visit the journal online (http://dx.doi.org/ 10.1136/10.1136/bmjopen2016-012879).

Received 29 May 2016 Revised 25 February 2017 Accepted 13 March 2017

CrossMark

School of Acupuncture and Tuina, Chengdu University of Traditional Chinese Medicine, Chengdu, China

Correspondence to

Dr Yong Tang;

tangyong@cdutcm.edu.cn

\section{ABSTRACT}

Introduction Knee osteoarthritis is a common form of arthritis in elderly patients that is characterised by pain and functional limitation. Moxibustion has been employed to relieve chronic pain as an alternative therapy for knee osteoarthritis. However, the evidence of its efficacy is equivocal due to the low methodological quality in most clinical studies. Therefore, we are performing a doubleblinded, double-placebo, randomised controlled trial to evaluate the efficacy of moxibustion in participants with knee osteoarthritis.

Methods and analysis This is a multicentre, double-blinded, double-placebo, randomised controlled clinical trial. 144 eligible participants with knee osteoarthritis will be randomly assigned to two different groups in a 1:1 ratio. Participants in the moxibustion group will undergo active moxibustion plus placebo gel, whereas participants in the control group will receive diclofenac sodium gel plus placebo moxibustion. Each participant will receive 12 sessions of active/placebo moxibustion at three acupoints (ST35, ST36 and EX-LE4) as well as 2 months of follow-up. Diclofenac sodium gel or placebo gel at a dose of $4 \mathrm{~g}$ per knee will be applied three times per day for 4 weeks. The primary outcome measure will be the Western Ontario and McMaster Universities Osteoarthritis Index (WOMAC) score change at the end of the intervention period from baseline. The secondary outcome measures include changes of other subscales (pain, stiffness and function) of WOMAC, visual analogue scale and patient globalassessment. The safety of moxibustion and diclofenac sodium gel will be assessed at every visit.

Ethics and dissemination This trial has been approved by the Sichuan Regional Ethics Review Committee (permission number: 2015KL-014). The results of this study are expected to provide clinical evidence on the efficacy of moxibustion for pain relief and physical function improvement in patients with knee osteoarthritis. The findings will be submitted for publication in peer-reviewed medical journals and presented at relevant academic conferences.

Trial registration number NCT02769572.

\section{BACKGROUND}

Osteoarthritis (OA) is the most common form of joint disease that most frequently affects
Strengths and limitations of this study

- This study is the first double-blinded, doubleplacebo, randomised controlled trial comparing moxibustion versus diclofenac sodium gel for pain relief and physical function improvement in participants with knee osteoarthritis.

- Methodological rigour, including adequate concealment of random allocation, strict quality control and the use of a valid placebo moxibustion device aim to reduce the risk of bias.

- A limitation of this study is that there is no long-term follow-up.

the knee joint. ${ }^{1}$ Pain and functional limitation are the primary clinical symptoms of knee OA that prevent patients from engaging in their usual activities. $^{2}$ Data have shown that $19.4 \%$ of people aged $>60$ years experience symptoms of knee OA. ${ }^{3}$ It is estimated that more than 20million Americans and 35-40 million Europeans suffer from OA. The currently recommended non-pharmacological and pharmacological treatments for OA aim to control pain and improve physical dysfunction. ${ }^{56}$ Oral non-steroidal anti-inflammatory drugs (NSAIDs) have become integral to the management of OA. However, these drugs have significant dose-related risks of gastrointestinal, cardiovascular and renal adverse events. ${ }^{7-10}$ As a first-line alternative medicine and a relatively new option for relieving pain due to knee OA, topical NSAIDs have shown equivalent efficacy compared with oral NSAIDs ${ }^{11}$ and have been known to minimise the risk of adverse events compared with oral NSAID exposure. ${ }^{12} \mathrm{~A}$ recent meta-analysis revealed that the administration of topical diclofenac is effective in providing pain relief as a treatment for OA, although improvements in physical function are unclear. ${ }^{13}$ Consequently, researchers have 


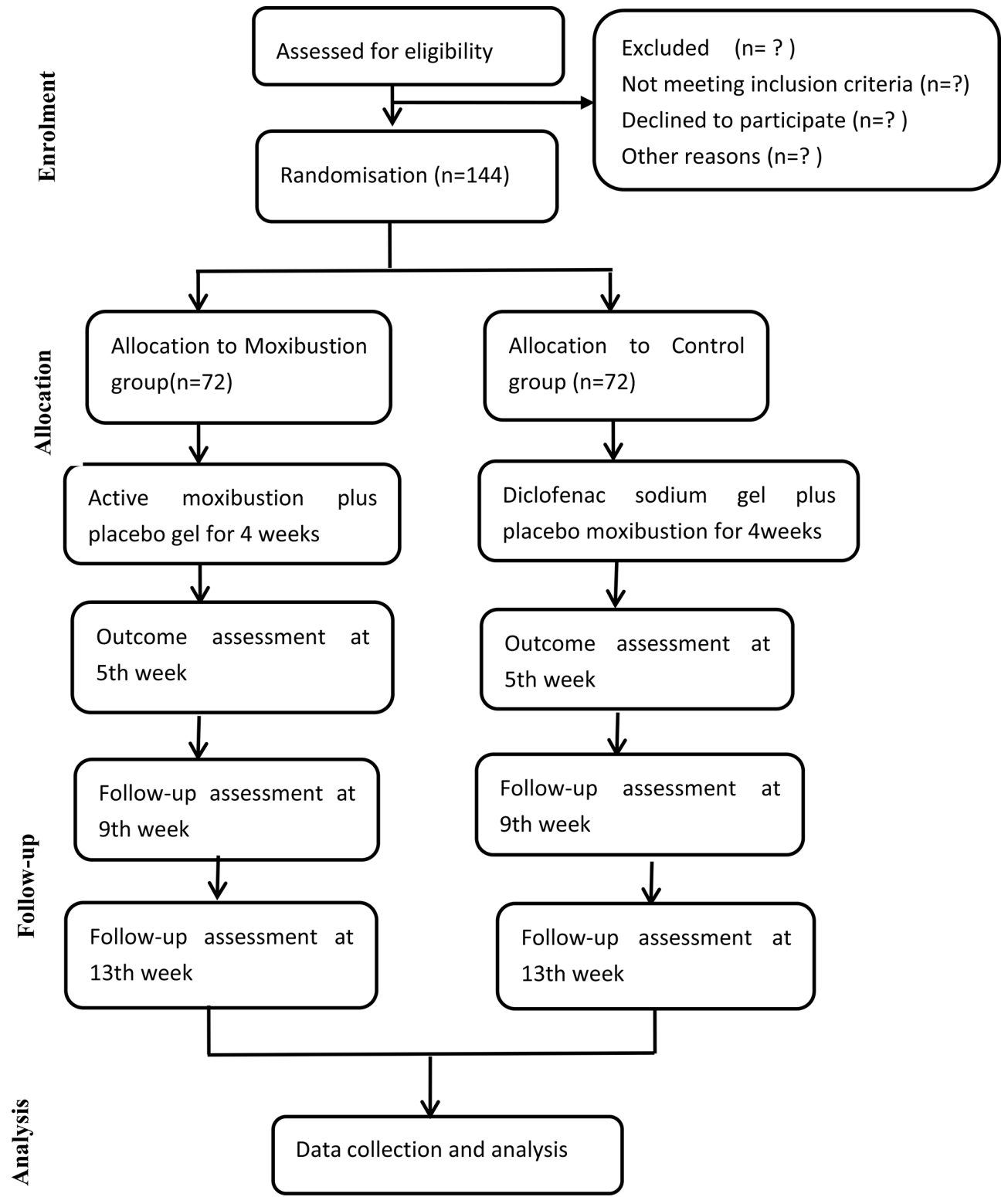

Figure 1 Flow chart of the study procedure.

gradually turned their attention to non-pharmacological therapies such as acupuncture and moxibustion. ${ }^{14}$

Moxibustion is a traditional oriental therapy that treats diseases through thermal stimulation by burning herbs, primarily Artemisia vulgaris, at specific spots on the skin. ${ }^{15}$ In the clinic, moxibustion is widely used to treat various diseases and their indications including OA. ${ }^{16}$ Clinical studies have shown that moxibustion is effective in treating knee OA. ${ }^{17-20}$ In a recent systematic review including 13 randomised controlled trials (RCTs) with a total of 1309 patients, the effects of moxibustion on the target population were found to be nearly equal to the effects of oral drug and intra-articular injection. ${ }^{21}$ However, the risk of bias was high in most of the included studies because of low methodological quality, owing to inappropriate control group setting and a lack of suitable double blinding methodology. So the role of moxibustion in knee OA is still a matter of controversy.
For these reasons, it is necessary to design a rigorous trial to evaluate the effectiveness of moxibustion in treating knee OA. So we decided to perform a randomised, double-blinded, double-placebo clinical study (online supplementary file) to investigate the efficacy of moxibustion in participants with knee OA.

\section{METHODS}

Study design

This is a multicentre, double-blinded, double-placebo, randomised controlled clinical study. The total duration of the trial will be 2 years, from May 2016 to July 2018. Fifteen clinical research centres in China will participate in this trial: Affiliated Hospital of Chengdu University of Traditional Chinese Medicine (TCM); Chengdu First People's Hospital; Sichuan Second Hospital of TCM; Sport Hospital Attached to Chengdu Sport Institute; Sichuan 
Orthopaedic Hospital; Pi Country People's Hospital; Xinqiao Hospital, Third Military Medical University; Xinjin Hospital of TCM; Medical Center Hospital of Qionglai City; Chongqing Traditional Chinese Medicine Hospital; Affiliated Hospital of Shaanxi University of Chinese Medicine; Xi'an Honghui Hospital; Nanjing Hospital of TCM; Affiliated Hospital of Nanjing University of TCM and Central Hospital of ZiBo. This protocol has been approved by the Sichuan Regional Ethics Review Committee (permission number 2015KL-014). Written consent will be obtained from each participant prior to enrolment. Eligible patients will be randomly assigned to the moxibustion group (active moxibustion plus placebo gel) or the control group (diclofenac sodium gel plus placebo moxibustion) in a 1:1 allocation ratio. The duration of the study period is 13 weeks, with a 1-week run-in period, 4-week treatment phase and 8-week follow-up phase. Outcome measurements will be evaluated at week 0 (baseline), week 2 , week 4 , week 8 and week 12 . The evaluation of participants and the analysis of the results will be performed by appointed professionals blinded to the group allocation. A flow chart of the trial procedure is presented in figure 1 .

\section{Recruitment}

Two strategies will be used to recruit participants with knee OA. First, participants will be recruited from the outpatient departments of the participating hospitals. Second, printed recruitment posters will be distributed in each participating hospital and nearby communities by researchers. The posters will contain a brief introduction about the trial, the details of free treatments offered to eligible participants and the contact information of the researcher. Participants who are interested in participating in this study can contact the researcher directly. The first participant was included on 24 May 2016.

\section{Participants}

\section{Inclusion criteria}

Participants will be included if they meet the following the criteria: (1) male or female gender, aged between 40 and 75 years and having knee OA diagnosed according to American College of Rheumatology criteria $^{5}$; (2) radiological confirmation of OA in one or both knees (Kellgren-Lawrence score of 2 or 3); (3) had knee pain for a duration of more than 3 months; (4) the average severity of knee pain being at least 3 points on a 10-point visual analogue scale (VAS); (5) willingness to be randomly assigned and comply with our study protocol; and (6) agreed to sign the consent form.

\section{Exclusion criteria}

Participants will be excluded from the study if they: (1) have pain in the knee that may be caused by inflammatory, malignant, autoimmune disease or traumatic injury; (2) have serious diseases including cancer, uncontrolled hypertension, diabetes mellitus requiring insulin injection; life-threatening cardiovascular or neurological events; chronic respiratory disease; bleeding disorders; clinically active renal, hepatic or peptic ulcer diseases and serious mental diseases; (3) have undergone knee replacement surgery or arthroscopy of the affected knee within the past year, or received steroid or hyaluronic acid injection in the knee joints within the previous 3 months; (4) have received physiotherapy including acupuncture or cupping for knee pain during the previous 4 weeks; (5) have had previous experience with moxibustion treatment; (6) are pregnant or lactating women; and (7) are participating in another clinical trial.

\section{Randomisation and allocation concealment}

Randomisation will be performed using a computerised random number generator operated by an independent statistician from the centre with no clinical involvement in this trial. The generated list of random numbers will be concealed using sequentially numbered, opaque, sealed envelopes. The participants' screening sequence numbers will be printed outside the envelope, whereas the group names will be printed inside. After a participant has met all selection criteria and completed the baseline assessments, the researcher will inform the statistician in the centre. The statistician will open the envelope according to the participant's screening sequence number and then assign the participant to either the moxibustion group or the control group.

\section{Blinding}

This is a double-blinded trial as the practitioners and the participants are both unaware of the assignments. The active moxibustion devices plus placebo gel will be placed together in one treatment room, whereas diclofenac sodium gel plus placebo moxibustion devices will be placed together in another treatment room. Each treatment room will have a designated practitioner to perform the active or placebo moxibustion. The study coordinator in every participating hospital, who will not be involved in treatment or assessment, will inform the participants about which treatment room they will need to go to receive moxibustion. Similarly, the outcome assessors and the data analysts will also be blinded to the allocation throughout the study.

\section{Interventions}

\section{Drug administration}

Participants in the control group will receive diclofenac sodium gel, while placebo gel will be administered to the moxibustion group. The placebo gel will be identical in composition to diclofenac sodium gel, except for the absence of diclofenac sodium, and have no counterirritant or other analgesic properties that might confound efficacy assessments. The two treatments will also be identical in appearance, smell and texture. Participants will be provided with detailed instructions for the use and application of the gel. 

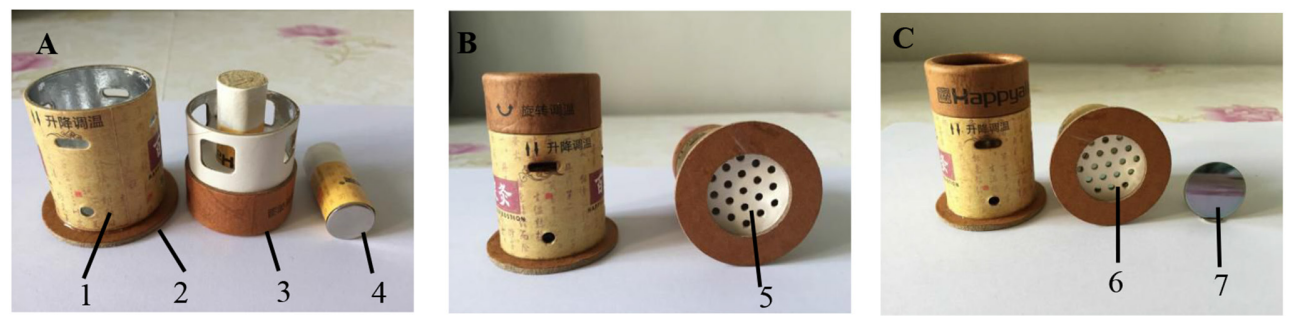

Figure 2 Diagram of the moxibustion device. (A) The components of the device: 1, cylindrical cardboard tube; 2, the base of the device with an adhesive membrane; 3, cylindrical cap; 4, moxa pillar with a magnet at the top. (B) The treatment moxibustion device: 5 , the bottom with holes to allow heat and smoke to radiate to the acupoints. (C) The placebo moxibustion device: 6 , the bottom with a thermal insulation panel to block smoke and heat; 7 , the thermal insulation board.

A dose of $4 \mathrm{~g}$ diclofenac sodium gel or placebo gel will be applied to the surface of each affected knee three times daily for 4 weeks. During a run-in period of 1 week and the treatment period, participants will refrain from using any NSAIDs or analgesics except for a 'rescue analgesic' (1-2 tablets of $500 \mathrm{mg}$ acetaminophen orally as needed, maximum $4 \mathrm{~g}$ /day), but the use of rescue medicine use will be recorded by participants in the their diaries.

\section{Moxibustion devices}

We will apply the novel moxibustion device that was invented by Professor Zhao of Beijing University of TCM (figure 2). This device is composed of three parts: a cylindrical cardboard tube, a cylindrical cap and a moxa pillar with magnet in the top; the base of the device is an adhesive membrane (figure 2A). The placebo moxibustion device resembles the active one in appearance, except the bottom of the device. The active moxibustion device has holes at the bottom to allow heat and smoke to radiate to the acupoints (figure 2B), but the placebo moxibustion device has a thermal insulation board over its bottom to isolate the smoke and most of the heat, preventing them from radiating to the skin (figure 2C). Reliability of this device was previously tested and validated by Zhao and colleagues. $^{22}$

\section{Moxibustion treatment procedure}

According to the theory of TCM and based on previous clinical trials, ${ }^{2324}$ we will select three standard acupuncture points: ST35, ST36 and EX-LE4. Practitioners will have at least 5 years of training in acupuncture and moxibustion. Three sessions per week will be performed during a 4-week period for a total of 12 sessions. During treatment, participants can choose to sit or lie down in a supine position with the affected knee(s) exposed. The active or placebo device will be affixed at each acupoint in the affected knee(s), then practitioners will twist off the cap and fix the moxa pillar to the cap. Once the moxa pillar is lit, the cap will be installed on the cylindrical cardboard tube. The distance between the burning moxa pillar port and skin is about $1 \mathrm{~cm}$. After burning, the residual pillar will be removed with tweezers, and another pillar will be inserted and lit. A moxa pillar burns for about $10 \mathrm{~min}$, and three consecutive moxa pillars will be burned at each point making each treatment session last about $30 \mathrm{~min}$.

Table 1 Time of visits and data collection

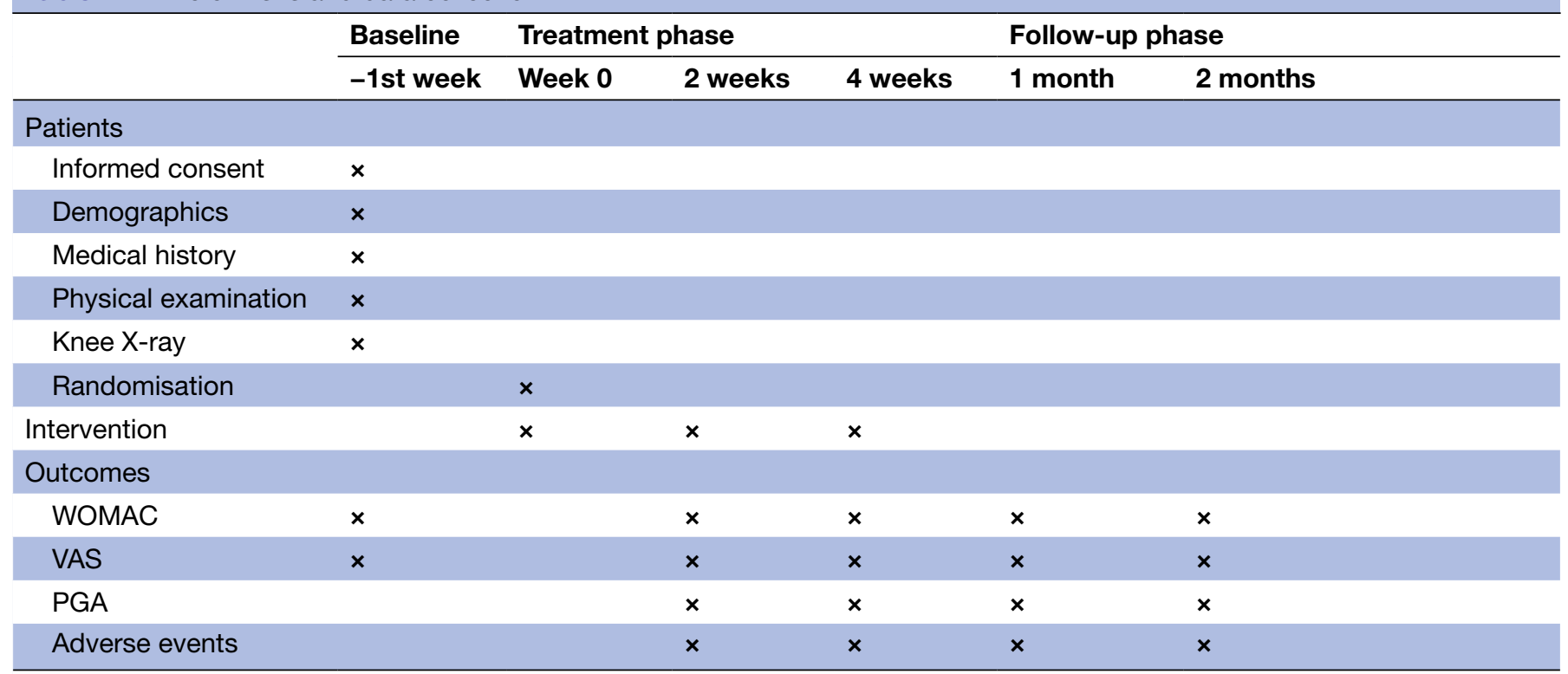

WOMAC, Western Ontario and McMaster Universities Osteoarthritis Index; VAS, visual analogue scale; PGA, patient global assessment. 


\section{Outcome measures}

\section{Primary outcome}

The primary outcome measure will be the mean change in the global scale value of the Western Ontario and McMaster Universities Osteoarthritis Index (WOMAC) from baseline to 4 weeks. The WOMAC is designed to measure dysfunction and pain associated with $\mathrm{OA}$; it includes three subscales to measure pain (five questions), stiffness (two questions) and physical function (17 questions) in knee OA, with higher scores indicating more severe impairment. ${ }^{25} 26$ The Likert version of the WOMAC rates each question on an ordinal scale of 0-4, with lower scores indicating lower levels of symptoms or physical disability. ${ }^{27}$ This method has been widely used in clinical trials to assess the OA of the knee or hip; ${ }^{28-30}$ if both knees were affected, the more severe knee will be assessed.

\section{Secondary outcomes}

The secondary outcomes include the mean changes in the WOMAC subscales (pain, stiffness and function) at 2, 4, 8 and 12 weeks from baseline. The pain intensity of knee OA will be assessed using the VAS, which is a $100 \mathrm{~mm}$ line ranging from 0 (no pain) to 100 (worst possible pain). ${ }^{31}$ We will evaluate the mean change in the VAS at 2, 4, 8 and 12 weeks from baseline. The patient global assessment is a single-item, patient-reported outcome measure to assess aspects of pain control other than pain intensity. ${ }^{32}$ Participants individually evaluate their improvement from baseline by selecting one of five options (much improved, minimally improved, no change, minimally worse or much worse) at 2, 4, 8 and 12 weeks.

\section{Adverse events}

Any adverse events (described as unfavourable or unintended signs, symptoms or diseases occurring after treatment) related to moxibustion and diclofenac sodium gel will be observed and reported by participants and practitioners during each visit. In addition, all vital signs and adverse events will be recorded in detail in the case report form (CRF) by the corresponding research staff. The outcome measurement time points are provided in detail in table 1.

\section{Quality control}

All practitioners, assessors and statisticians will receive training uniformly prior to the trial to guarantee the use of consistent practices among the 15 clinical research centres. The training programme includes training on diagnosis of knee OA, inclusion and exclusion criteria, standard operation procedures, observation time, outcome measures, the completion of CRFs, locations of the acupoints and data processing of moxibustion manipulation techniques throughout the study. Treatment and placebo moxibustion will be performed by acupuncturists with at least 5 years of training in acupuncture and moxibustion. The study will be regularly monitored by clinical monitors to ensure the quality of the trial.

\section{Data management and monitoring}

Data collection will be performed at baseline and at 2, 4, 8 and 12 weeks after the intervention for the moxibustion group and the control group. A data managing and monitoring board will be set up to supervise data acquisition and entry. Adverse events or unintended effects of moxibustion or diclofenac sodium gel will first be recorded in the CRFs by physicians who are in charge, then the physicians will call the data monitoring centre within 24 hours. Collected data will be entered into electronic CRFs (eCRFs) with double entry. The eCRFs will be uploaded to a central server. The principal investigators will have access to the data. In principle, clinical information will not be released without the permission of the principal investigator, with the exception of an emergency or as necessary for monitoring and auditing by the data monitoring committee (DMC). The DMC comprises an orthopaedist, a medical statistician and an epidemiologist, who is independent of the investigators and sponsors. The role of the DMC is to audit the trial conducted every month after the trial starts and write monthly reports on recruitment of participants, compliance of participants to the assigned treatment and whether the CRFs are correctly filled in.

\section{Sample size}

The primary efficacy parameter is the decrease of WOMAC score from baseline to the end of treatment after 4 weeks. According to the previous literature and our pilot study, we assume a reduction of $36 \%$ in the WOMAC score to be effective. ${ }^{33} 34$ The statistical power is 0.90 , and the significance level is 0.05 ; it is estimated that the dropout rate is $20 \%$; the sample size will be $\mathrm{n}=72$ for each group (total $\mathrm{n}=144)$.

\section{Statistical analysis}

The data analysis will be performed by an independent statistician using the Statistical Package for the Social Sciences V.19.0 statistical software package. Significant levels will be set at $\mathrm{p}<0.05$. All analyses will be based on the intention-to-treat principle. Missing values will be handled by the modern imputation methods which will be accomplished using a set of repeated imputations created by predictive models based on the majority of participants with complete data. Demographic characteristics and other baseline values will be described using descriptive statistics for each group. Continuous variables with normal distribution will be expressed as the means with SD; for abnormally distributed variables, the data will be expressed as medians with a centile range (such as the 25 th and 75 th centiles). Numbers and proportions will be used to describe the categorical variables. Between-group differences in primary and secondary outcomes will be tested using repeated measures analyses of variance. Considering that the efficacy might be affected by factors such as age, degree of disease and course of disease, all factors will be considered as covariates for covariance analysis. 


\section{ETHICS AND DISSEMINATION \\ Ethics}

This trail is conducted in accordance with the Declaration of Helsinki. Ethics approval has been obtained from the Sichuan Regional Ethics Review Committee (permission number: 2015KL-014). Before the study, trained research assistants will have a full discussion about the potential benefits and risks of this study with participants. Then the research assistants will obtain written informed consent from participants willing to participate in the trial. Any modifications to the protocol will be reported and approved by the Sichuan Regional Ethics Review Committee and will be communicated with the trial registry, investigators and data monitoring researchers.

\section{Dissemination}

The study results will be disseminated via conference presentations and papers published in academic peer-reviewed journals. The participants, healthcare professionals, the public and other relevant groups will also be informed of the study results.

\section{Confidentiality}

In order to protect confidentiality before, during and after the trial, all investigators will always maintain a strict privacy policy. Participants' information will be stored in locked file cabinets at the study sites with limited access; only investigators have the right to access the data. After the trial, all paper and electronic versions of the CRFs will be preserved in the secure research archives at Chengdu University of TCM for 10 years and will only be viewed by the research team. Participants will also have the right to know their personal data and the final study results.

\section{DISCUSSION}

The objective of this study is to evaluate the effectiveness and safety of moxibustion for treating knee OA. The highlight of this trial is that the double-blinded, double-placebo design has been used for the first time in moxibustion clinical trials. Adequate concealment of random allocation, strict quality control and the use of a valid placebo moxibustion device are the strengths of this study. A limitation of this study is that there is no long-term follow-up because the short-term superiority of moxibustion over diclofenac sodium gel for the treatment of knee OA has not been confirmed.

Double-blinding is a crucial aspect of study design for accurately measuring the efficacy of any treatment in a clinical trial, where both participant and practitioner are masked to the treatment condition. ${ }^{35}$ In high-quality RCTs, placebo control is always adopted to maintain the blinding method. In this study, we will adopt the double-placebo design for placebo moxibustion in the control group, while the placebo gel will be applied to the moxibustion group to achieve double-blinding. This design is an important methodology for reducing bias that was first introduced in a clinical trial to compare indomethacin and phenylbutazone for the treatment of a variety of rheumatic diseases in the $1960 \mathrm{~s} .{ }^{36}$ From then on, the double-placebo design was widely used to compare drugs that were administered by different routes in the pharmaceutical drug trials. ${ }^{37}{ }^{38}$ In acupuncture clinical trials, Wang et $a l^{39}$ first applied this design to evaluate the efficacy of acupuncture for migraine prophylaxis. However, the double-placebo design is seldom used to assess the effect of moxibustion compared with drugs or other physical therapies in treating knee OA in previous studies. So, we adopted this rigorous design to compare the efficacy of moxibustion and diclofenac sodium gel in treating $\mathrm{OA}$ of the knee.

In conclusion, the results of this trial will help to provide evidence on the efficacy of moxibustion for pain relief and physical function improvement in patients with knee OA. It is also expected to provide reliable raw data for systematic review and evidence-based medicine.

Contributors J-YZ, LL, L-LZ contributed equally to this work. J-YZ, YT, S-GY conceived this trial. QFW, H-YY, C-SZ, PL, J-XP participated in the design of this trial. $\mathrm{J}-\mathrm{YZ}, \mathrm{LL}, \mathrm{L}-\mathrm{LZ}$ drafted the first version of the manuscript for this trial. All authors contributed to the further writing of the manuscript in addition to reading and approving the final version of the manuscript.

Funding This work is supported by grants from the National Basic Research Program of China (973 Program, 2015CB554504), Sichuan Provincial Innovative Research Team Program (2014TD0018, 2015TD0010) and Innovative Research Team in University of Sichuan Province (16TD0015).

Competing interests None declared.

Patient consent Obtained.

Ethics approval The ethics review boards of the Sichuan Regional Ethics Review Committee.

Provenance and peer review Not commissioned; externally peer reviewed.

Data sharing statement The results of this pilot study will be disseminated via peer-reviewed publications and conference presentations. All of the data are available.

Open Access This is an Open Access article distributed in accordance with the Creative Commons Attribution Non Commercial (CC BY-NC 4.0) license, which permits others to distribute, remix, adapt, build upon this work non-commercially, and license their derivative works on different terms, provided the original work is properly cited and the use is non-commercial. See: http://creativecommons.org/ licenses/by-nc/4.0/

(C) Article author(s) (or their employer(s) unless otherwise stated in the text of the article) 2017. All rights reserved. No commercial use is permitted unless otherwise expressly granted.

\section{REFERENCES}

1. Creamer P, Hochberg MC. Osteoarthritis. Lancet 1997;350:503-9.

2. Felson DT. Osteoarthritis of the knee. New Engl J Med 2006;354:841-8.

3. Xiang YJ, Dai SM. Prevalence of rheumatic diseases and disability in China. Rheumatol Int 2009;29:481-90.

4. Mobasheri A. The future of osteoarthritis therapeutics: targeted pharmacological therapy. Curr Rheumatol Rep 2013;15:1-13.

5. Gamble R, Wyeth-Ayerst J, Johnson EL. Recommendations for the medical management of osteoarthritis of the hip and knee: 2000 update. American College of Rheumatology Subcommittee on Osteoarthritis Guidelines. Arthritis Rheum 2000;43:1905-15.

6. Jordan KM, Arden NK, Doherty M, et al; Standing Committee for International Clinical Studies Including Therapeutic Trials ESCISIT. EULAR Recommendations 2003: an evidence based approach to the management of knee osteoarthritis: report of a task force of the Standing Committee for International Clinical Studies Including Therapeutic Trials (ESCISIT). Ann Rheum Dis 2003;62:1145-55.

7. Silverstein FE, Faich G, Goldstein JL, et al. Gastrointestinal toxicity with celecoxib vs nonsteroidal anti-inflammatory drugs 
for osteoarthritis and rheumatoid arthritis: the CLASS study: a randomized controlled trial. JAMA 2000;284:1247-55.

8. Wolfe MM, Lichtenstein DR, Singh G. Gastrointestinal toxicity of nonsteroidal antiinflammatory drugs. N Engl J Med 1999;340:1888-99.

9. Whelton A. Nephrotoxicity of nonsteroidal anti-inflammatory drugs: physiologic foundations and clinical implications. Am J Med 1999;106:13S-24S.

10. Antman EM, Bennett JS, Daugherty A, et al; American Heart Association. Use of nonsteroidal antiinflammatory drugs: an update for clinicians: a scientific statement from the American heart association. Circulation 2007;115:1634-42.

11. Zhang W, Nuki G, Moskowitz RW, et al. OARSI recommendations for the management of hip and knee osteoarthritis: part III: changes in evidence following systematic cumulative update of research published through January 2009. Osteoarthritis Cartilage 2010;18:476-99.

12. Altman RD, Dreiser RL, Fisher $\mathrm{CL}$, et al. Diclofenac sodium gel in patients with primary hand osteoarthritis: a randomized, doubleblind, placebo-controlled trial. J Rheumatol 2009;36:1991-9.

13. Deng ZH, Zeng C, Yang Y, et al. Topical diclofenac therapy for osteoarthritis: a meta-analysis of randomized controlled trials. Clin Rheumatol 2016;35:1253-61.

14. Chen R, Chen M, Su T, et al. Heat-sensitive moxibustion in patients with osteoarthritis of the knee: a three-armed multicentre randomised active control trial. Acupunct Med 2015;33:262-9.

15. Cheng CW, Fu SF, Zhou QH, et al. Extending the CONSORT statement to moxibustion. J Integr Med 2013;11:54-63.

16. Huang Q-feng, Wu H-gan, Liu J, et al. Bibliometric analysis of diseases spectrum of moxibustion therapy. J Acupunct Tuina Sci 2012;10:342-8

17. Zhou ZL, Sun K, Cheng HL, et al. Observations on the therapeutic effect of herbal cake-separated moxibustion on knee osteoarthritis of blood stasis type. Shanghai J Acup Moxi 2010;29:45-7.

18. Sun K, Yang J, Shen DK. Clinical observation on treatment of primary knee osteoarthritis of liver and kidney deficiency type with aconite cake-separated moxibustion. Zhongguo Zhen Jiu 2008;28:87-90.

19. Cheng HL, Han W, Pj H, et al. Clinical study of differential selection of objects separated moxibustion for treatment of knee osteoarthritis. Clin J Tradit Chin Med 2008;20:114-16.

20. Kim TH, Kim KH, Kang JW, et al. Moxibustion treatment for knee osteoarthritis: a multi-centre, non-blinded, randomised controlled trial on the effectiveness and safety of the moxibustion treatment versus usual care in knee osteoarthritis patients. PLoS One 2014:9:e101973.

21. Song GM, Tian $\mathrm{X}$, Jin $\mathrm{YH}$, et al. Moxibustion is an alternative in treating knee osteoarthritis: the evidence from systematic review and meta-analysis. Medicine (Baltimore) 2016;95:e2790.

22. Zhao B, Wang X, Lin Z, Liu R, et al. A novel sham moxibustion device: a randomized, placebo-controlled trial. Complement Ther Med 2006;14:53-60.

23. Tian C, Lu J, Cao LD. Analysis of acupoint selecting regularity treating Knee-bone arthritis in resent 5 years. Modern Traditional Chinese Medicine 2013;33:70-3.
24. Zhang $\mathrm{H}$, Zhao BX, Song $\mathrm{Y}$, et al. Analysis of regularities in point selection and clinical study of moxibustion for knee osteoarthritis in recent 10 years. Information on Traditional Chinese Medicine 2015;32:118-20.

25. Bellamy N, Buchanan WW, Goldsmith $\mathrm{CH}$, et al. Validation study of WOMAC: a health status instrument for measuring clinically important patient relevant outcomes to antirheumatic drug therapy in patients with osteoarthritis of the hip or knee. J Rheumatol 1988; 15:1833-40.

26. Wolfe F. Determinants of WOMAC function, pain and stiffness scores: evidence for the role of low back pain, symptom counts, fatigue and depression in osteoarthritis, rheumatoid arthritis and fibromyalgia. Rheumatology 1999;38:355-61.

27. McConnell S, Kolopack P, Davis AM. The Western Ontario and McMaster Universities Osteoarthritis Index (WOMAC): a review of its utility and measurement properties. Arthritis Rheum 2001;45:453-61.

28. Hinman RS, McCrory P, Pirotta M, et al. Acupuncture for chronic knee pain: a randomized clinical trial. JAMA 2014;312:1313-22.

29. Scharf HP, Mansmann U, Streitberger K, et al. Acupuncture and knee osteoarthritis: a three-armed randomized trial. Ann Intern Med 2006:145:12-20.

30. Baraf HS, Gold MS, Clark MB, et al. Safety and efficacy of topical diclofenac sodium $1 \%$ gel in knee osteoarthritis: a randomized controlled trial. Phys Sportsmed 2010;38:19-28.

31. Carlsson AM. Assessment of chronic pain. Aspects of the reliability and validity of the visual analogue scale. Pain 1983;16:87-101.

32. Rothman M, Vallow S, Damaraju CV, et al. Using the patient global assessment of the method of pain control to assess new analgesic modalities in clinical trials. Curr Med Res Opin 2009;25:1433-43.

33. Altman R, Asch E, Bloch D, et al. Development of criteria for the classification and reporting of osteoarthritis: classification of osteoarthritis of the knee. Arthritis Rheum 1986;29:1039-49.

34. Goldsmith $\mathrm{CH}$, Boers M, Bombardier $\mathrm{C}$, et al. Criteria for clinically important changes in outcomes: development, scoring and evaluation of rheumatoid arthritis patient and trial profiles. OMERACT Committee. J Rheumatol 1993;20:561-5.

35. Takakura N, Takayama M, Kawase A, et al. Double blinding with a new placebo needle: a further validation study. Acupunct Med 2010;28:144-8

36. Maru i A, Feren i SF. Adoption of the double dummy trial design to reduce observer bias in testing treatments. $J R$ Soc Med 2013:106:196-8.

37. Lavine JE, Schwimmer JB, Van Natta ML, et al; Nonalcoholic Steatohepatitis Clinical Research Network. Effect of vitamin E or metformin for treatment of nonalcoholic fatty liver disease in children and adolescents: the TONIC randomized controlled trial. JAMA 2011;305:1659-68

38. Olanow CW, Kieburtz K, Odin P, et al. Double-blind, double-placebo, randomized study of continuous intrajejunal infusion of levodopacarbidopa intestinal gel in advanced Parkinson's disease. Lancet Neurol 2014;13:141-9.

39. Wang LP, Zhang XZ, Guo J, et al. Efficacy of acupuncture for migraine prophylaxis: a single-blinded, double-dummy, randomized controlled trial. Pain 2011;152:1864-71. 